\title{
Breast Carcinoma Grade
}

National Cancer Institute

\section{Source}

National Cancer Institute. Breast Carcinoma Grade. NCI Thesaurus. Code C147915.

A modification of the Notting ham grading system for use in grading breast carcinoma. 